\title{
Isolated Subscapularis Tendon Tear in a Skeletally Immature Soccer Player
}

\author{
Paolo Avanzi ${ }^{1}$ Luca Dei Giudici ${ }^{2}$ Roberto Giovarruscio ${ }^{1}$ Antonio Gigante ${ }^{2}$ Claudio Zorzi ${ }^{1}$ \\ 1 Division of Orthopaedics, Ospedale Sacro Cuore-Don Calabria, \\ Address for correspondence Luca Dei Giudici, MD, Clinical \\ Negrar, Verona, Italy \\ ${ }^{2}$ Clinical Orthopaedics, Università Politecnica delle Marche, \\ DISCLIMO, School of Medicine, Ancona, Italy \\ Orthopaedics, Università Politecnica delle Marche, DISCLIMO, School \\ of Medicine, Via Tronto, 10/A, Ancona 60126, Italy \\ (e-mail: lucadeigiudici@gmail.com).
}

Joints 2018;6:68-70.

\begin{abstract}
Subscapularis injury in adolescents, usually associated to an avulsion fracture of the lesser humeral tuberosity, accounts for less than $2 \%$ of all fractures of the proximal humerus. Isolated tears of the subscapularis tendon without a history of dislocation and associated avulsion fractures are an even rarer occurrence, and treatment is controversial. This article describes a rare case of a 12-year-old suffering from an isolated subscapularis tear and discusses its management. The patient was evaluated at presentation, and at 1 to 2.5 months after he underwent a cuff tear arthroscopic repair with a single "all suture" anchor loaded with two wires, active/passive range of motion (A/PROM), Constant-Murley score, and American Shoulder and Elbow Surgeons (ASES) score were noted. Patient reported an excellent outcome, recovered the whole ROM, was pain free, and returned to the previous

Keywords

- subscapularis

- shoulder

- arthroscopy

- adolescent level of activity. Isolated avulsion of the subscapularis tendon requires a high index of suspicion for a proper diagnosis as early treatment is required for a good recovery. Arthroscopy reserves more advantages in proper hands, restoring the previous levels of function and activity. An increase in attention for this condition is mandatory in a society where many adolescents are getting more and more active in high levels of sport activities.
\end{abstract}

\section{Introduction}

Rotator cuff injuries in adolescents are rare events, involving less than $1 \%$ of patients younger than 20 years. $^{1}$ When a subscapularis injury is present, it is associated to an avulsion fracture of the lesser humeral tuberosity, which accounts for less than $2 \%$ of all fractures of the proximal humerus, ${ }^{2}$ related to the proximal physis depicting a condition known as "little league shoulder." The scarcity of publications about this matter ${ }^{3}$ shows how isolated tear of the subscapularis tendon without a history of dislocation and associated avulsion fractures is the rarest occurrence of all. ${ }^{4}$

This article describes the rare case of a young adolescent athlete suffering from an isolated subscapularis tear, and discusses its management.

\section{Case Report}

A 12 years old, right-hand dominant, boy [ $154 \mathrm{~cm}, 48 \mathrm{~kg}$, body mass index (BMI) 20.24] presented at the office. He was a soccer

received

June 15, 2017

accepted after revision

October 25, 2017

published online

December 11, 2017 goalkeeper in a national professional league team, and reported a sudden onset of localized anterior shoulder pain 6 days earlier, right after a quarrel with an opponent that forced his arm in an excessive abduction and posterior traction. He referred persistent pain during rotations, overhead activities, and while holding his school bag. No dislocation or subjective shoulder instability were reported, nor a sensation of a pop or a snap. Clinical examination showed the following: active range of motion (AROM) was limited by anterior pain, at $45^{\circ}$ in external rotation with arm on the side and at $60^{\circ}$ with arm abducted at $90^{\circ}$; it was limited in internal rotation with the hand reaching the lumbar vertebras; it was limited in elevation at $115^{\circ}$. Passive ROM (PROM) was complete, apart from painful external rotation at $75^{\circ}$ with arm on the side and $85^{\circ}$ with arm abducted, and internal rotation that could not reach the dorsal spine. A weak iteratively reweighted least squares (IRLS) was noted, Bear Hug and Napoleon tests were painful and mildly weak; other special were negative. Differential diagnosis included, at this stage, a transitional fracture involving the anterior and anterosuperior rotator cuff, a bony Bankart lesion,
DOI https://doi.org/

10.1055/s-0037-1608952.

ISSN 2282-4324.
Copyright @ 2018 Georg Thieme Verlag

KG Stuttgart · New York
License terms

$\circledast(1) \Theta$ 

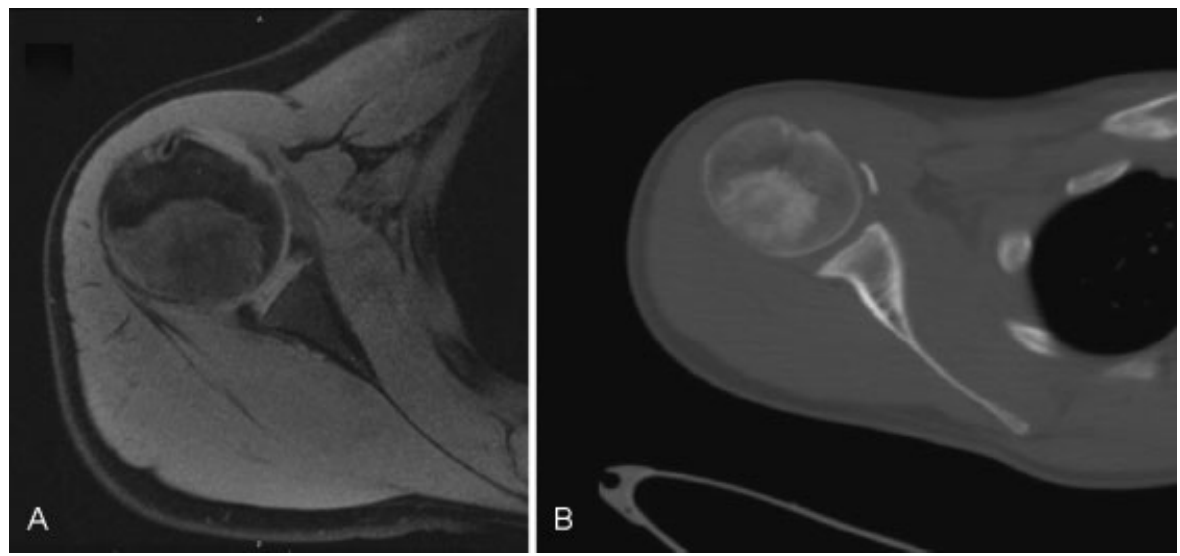

Fig. 1 (A) Magnetic resonance imaging (MRI) imaging of the patient's affected shoulder, a T2-weighted section. It is notable that the inhomogeneous signal from the subscapularis tendon and the integrity of the glenoid and the labrum, and intact lesser humeral tuberosity. (B) A computed tomography (CT) scan of the patient's affected shoulder. The cortical foil is clearly observable in the context of the subscapularis tendon.

a tear of the subscapularis tendon, and a humeral avulsion of the glenohumeral ligament (HAGL) lesion, therefore an X-ray study [anteroposterior (AP), lateral, and arch views] and an magnetic resonance imaging (MRI) were prescribed. No pathological signs were noted in the X-rays beside a little anterior opacity referred to as a calcification. MRI showed an inhomogeneous signal at the subscapularis insertion in the absence of lesser tuberosity fracture; MRI report described, instead, mild tendinosis of the supraspinatus and integrity of the remaining structures, advising for another II level study to clarify whether a doubtful signal from the glenoid was to interpret as a fracture or as closing physis. A computed tomography (CT) imaging with Population, Intervention, Comparison and Outcome (PICO) protocol was performed, and showed integrity of the right glenoid (with an area of $490 \mathrm{~mm}^{2}$, slightly bigger than the one of the unaffected shoulder), and a calcific foil $(1.5 \times 2 \mathrm{~mm})$ along the inner layers of the subscapularis, reported as a dystrophic calcification of a previous hematoma.

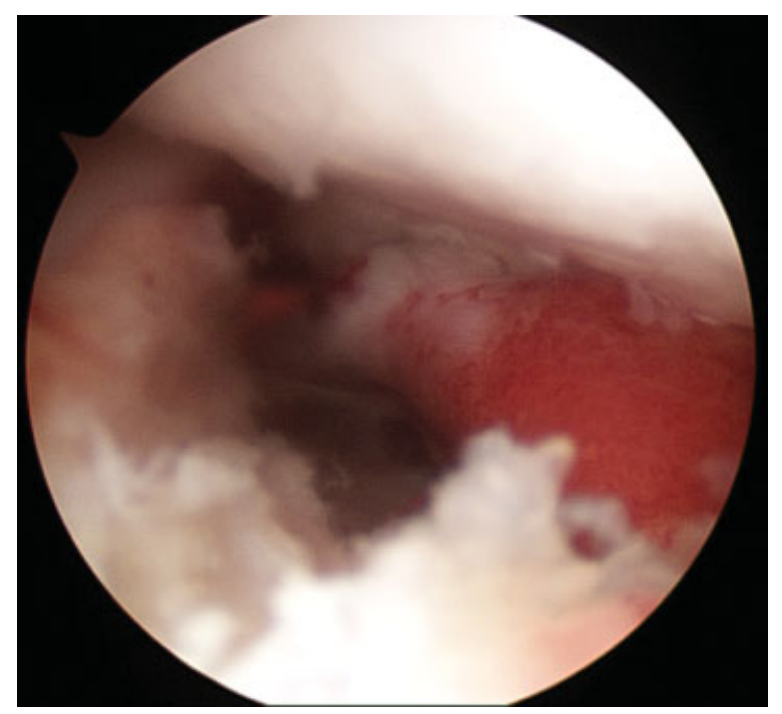

Fig. 2 Arthroscopic intraoperative view acquired at the end of the procedure; the reattached tendon after the suturing demonstrates a correct tensioning and a proper anatomical positioning.
Imaging is provided in - Fig. 1. Surgery was performed 29 days after trauma. An arthroscopy was performed in the lateral position, and a tear of the subscapularis tendon was confirmed, along with a mild synovitis. The rotators interval was opened, without any release of the bicipital groove and without bicep's tenotomy, and the subscapularis was released from capsular adhesions; the bony fragment was identified and removed with a shaver. Final step was the refreshing of the distal tendon border and his footprint on the lesser tuberosity, where an "all suture" anchor loaded with two FiberWire sutures (Y-Knot RC, ConMed, Utica, New York, United States) was used to reattach the tendon (-Fig. 2). Patient was discharged the day after surgery with a standardized protocol. Postoperative evaluations were performed at 1 week, 1,3 , and 5 months. At the latest follow-up, the patient was pain free and had full A/PROM; rotator cuff tests were negative (-Fig. 3), American Shoulder

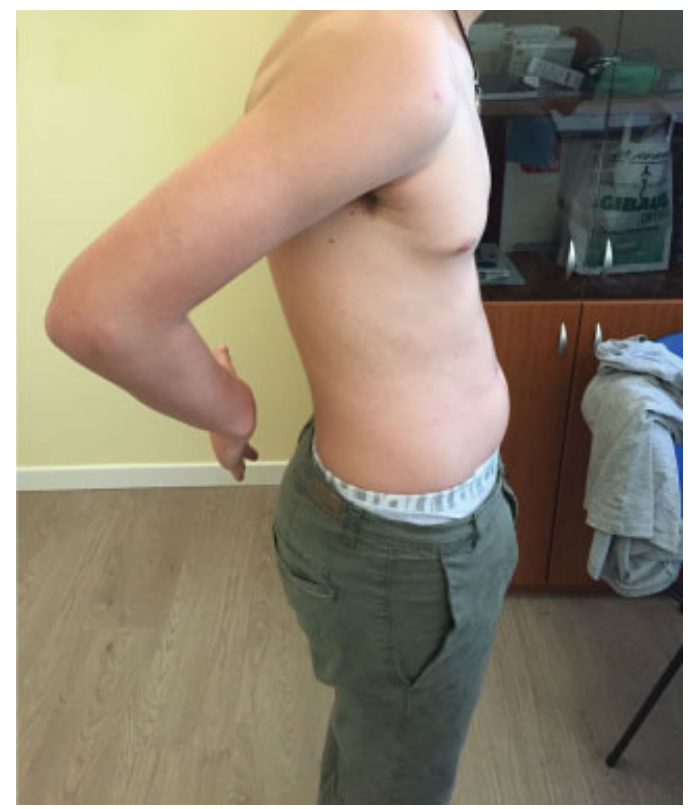

Fig. 3 Patient during the clinical examination at the latest follow-up: Lift-off test sign is now negative. 
and Elbow Surgeons (ASES) score was 94.99, and ConstantMurley score was 94, showing an increase of 43.33 and 24 point, respectively.

\section{Discussion}

The rare incidence of rotator cuff tears in adolescents is due to higher strength, elasticity, and resilience of their tissues, ${ }^{1}$ responsible for the usual association with avulsion fractures. Sudden abduction and external rotation is the mechanism reported for a subscapularis involvement; to resist the muscle exerts a forceful eccentric contraction, resulting in the avulsion of the lesser tuberosity or in an isolated tendon tear. ${ }^{2}$ Surgery seems an appropriate management ${ }^{3}$ in acute or subacute settings. Late diagnosis or nonoperative treatments could lead to the worsening of the mechanical dysfunction, disuse atrophy with fatty degeneration, and muscle retraction, and the need for a more complex surgery with inevitably worse outcomes and limitations. ${ }^{2}$ Literature provides only 60 cumulative cases, published since $1985,{ }^{3}$ therefore clear indications about the best treatment option are not available. The only seven similar cases ever published ${ }^{3}$ and treated arthroscopically demonstrated good outcomes, slightly inferior to an arthroscopic and open approach, and superior to the open repairs.

This article described one of the rarest happening after a trauma to the shoulder in an adolescent patient: an isolated subscapularis tendon tear. Clinical outcome was excellent due to some key factors, first of all early surgery. Arthroscopy was preferred over open repairs, to perform a less invasive procedure with minimal postoperative pain, and a rapid progression to full recovery, with a short-term return to play. ${ }^{5}$ Moreover, the avulsed tendon was fixated with "soft" hardware made solely of suture. This choice was possible because enough stability for the all-suture anchors was achieved, giving the patient a hardware-free repair that will eventually not influence physis physiology and will give a full range possibilities in the unfortunate event of a new shoulder surgery.

Lastly, some concerns could arise regarding the tendon of the long head of the bicep; in an adult patient, in fact, authors would prefer to proceed with a tenotomy. Considering the young age of the patient, and that the superficial portion of the pulley was not involved, the authors preferred to perform an articular subscapularis repair leaving the biceps tendon intact.

\section{Conflict of Interest}

None.

\section{References}

1 Tarkin IS, Morganti CM, Zillmer DA, McFarland EG, Giangarra CE. Rotator cuff tears in adolescent athletes. Am J Sports Med 2005;33 (04):596-601

2 Goeminne S, Debeer P. The natural evolution of neglected lesser tuberosity fractures in skeletally immature patients. J Shoulder Elbow Surg 2012;21(08):e6-e11

3 Vavken P, Bae DS, Waters PM, Flutie B, Kramer DE. Treating subscapularis and lesser tuberosity avulsion fractures in skeletally immature patients: a systematic review. Arthroscopy 2016; 32(05):919-928

4 Piasecki DP, Nicholson GP. Tears of the subscapularis tendon in athletes-diagnosis and repair techniques. Clin Sports Med 2008; 27(04):731-745

5 Heyworth BE, Dodson CC, Altchek DW. Arthroscopic repair of isolated subscapularis avulsion injuries in adolescent athletes. Clin J Sport Med 2008;18(05):461-463 